\title{
Report
}

\section{Functional connectivity of the posterior hippocampus is more dominant as we age}

\author{
Sonja Blum ${ }^{1}$, Christian Habeck ${ }^{2}$, Jason Steffener ${ }^{2}$, Qolamreza Razlighi ${ }^{2}$, \\ and Yaakov Stern ${ }^{2}$
}

${ }^{1}$ Neurology Department, New York University, New York, USA

${ }^{2}$ Neurology Department, Columbia University, New York, USA

\begin{abstract}
The role of the hippocampus in memory is dependent on its interaction with distributed brain areas. Anterior and posterior hippocampus have different roles in memory processing, and are impacted differently by aging in terms of structural decline, however, functional connectivity of these hippocampal regions in aging is not well understood. Young (age 17-30) and aging (age 60-69) cognitively normal subjects underwent resting-state functional MRI revealing a shift from anterior hippocampus dominant hippocampus connectivity in younger age group to posterior hippocampus dominant connectivity in aging subjects. We identified a subset of neocortical regions that are connected to the anterior hippocampus in younger adults but to the posterior hippocampus among older subjects, suggesting an age-related reorganization of hippocampal networks supporting normal cognitive function. We also performed volumetric analysis which revealed no significant structural differences between groups. These findings provide evidence that "functional anatomy" which supports normal memory performance changes across the life span.
\end{abstract}

Keywords: Memory; Hippocampus; Network.

The dynamic interaction between the hippocampus and neocortical regions is critical for memory functions (Dash, Hebert, \& Runyan, 2004; Marr, 1971; McClelland, McNaughton, \& O’Reilly, 1995; Nadel \& Moscovitch, 1997; Squire \& Alvarez, 1995; Teyler \& DiScenna, 1985). Animal and human studies suggest that anterior and posterior regions of the hippocampus form functionally distinct "circuits," and animal studies have demonstrated that the anterior and posterior hippocampus have distinct anatomical connections to other brain regions (for review, see Manns \& Eichenbaum, 2006; Moser \& Moser, 1998; Small, Schobel, Buxton, Witter, \& Barnes, 2011). Anterior hippocampus is tightly connected to the lateral entorhinal and perirhinal cortices and receives input from the amygdala, primary/unimodal sensory cortices, granular insular and orbitofrontal cortices, whereas the posterior hippocampus receives its primary input via the medial entorhinal and postrhinal/parahippocampal cortices, from polymodal association cortices including posterior parietal, visual association/V4, retrosplenial and superior temporal gyrus (Manns \& Eichenbaum, 2006; Moser \& Moser, 1998; Suzuki \& Amaral, 1994).

Kahn and colleagues (Kahn, Andrews-Hanna, Vincent, Snyder, \& Buckner, 2008) have reported anterior-posterior hippocampus functional connectivity differences in young human subjects using resting state BOLD fMRI, and the findings were relatively consistent with the expectations based on the animal literature.

Correspondence should be addressed to: Sonja Blum, Neurology Department, New York University. E-mail: Sonja.blum@nyumc.org

This work was supported by a grant from the National Institute of Aging (RO1AG26158) awarded to Yaakov Stern. Sonja Blum was supported by the Charles and Ann Lee Saunders Brown Fellowship.

(C) 2014 Taylor \& Francis 
Poppenk and Moskovitch (2011) further strengthen the claim that anterior and posterior hippocampus form functionally distinct regions by showing, again in young subjects, that recollection of memory depends on the posterior but not anterior hippocampus, and that the relationship of posterior hippocampus to memory is mediated by the functional connectivity of the hippocampus to neocortical regions.

Based on the anatomical differences in connectivity of the hippocampus along its anteriorposterior axis, evidence from animal research, as well as the evidence from functional brain imaging in young human subjects, we predicted that there is a distinct network of brain regions functionally connected with the anterior hippocampus and another network connected preferentially with the posterior hippocampus. In aging, the anterior hippocampus atrophies before the posterior (Chen, Chuah, Sim, \& Chee, 2010; Jack et al., 1997; Rajah, Kromas, Han, \& Pruessner, 2010). In this study, we explored the hypothesis that, in older subjects, the functional connectivity of the anterior hippocampus may be diminished, with posterior hippocampal functional connectivity compensating with increased connectivity with neocortical regions in the elderly.

We used resting state BOLD fMRI (Fox \& Raichle, 2007) to examine the functional connectivity of the anterior and posterior hippocampus in cognitively intact young and older human subjects. We also performed manual segmentation of hippocampi to measure total hippocampal volume, as well as anterior and posterior segment sizes in our sample of subjects.

\section{METHODS}

\section{Participants}

Twenty-four young participants (11 Male, mean age: 25.3 years, $S D: 2.8$ years), and 21 older participants (8 Male, mean age: 65.4 years, $S D: 2.5$ years) were recruited through random market mailing from within 10 miles of the Columbia University Medical Center. This provides a more representative population sample, as compared to recruiting within a university. Participants were screened to exclude individuals with a history of neurologic or psychiatric conditions and those using psychoactive medications. All participants were compensated for participation. Informed consent was obtained prior to testing under supervision of the Columbia University Medical Center Institutional Review Board. Demographic and baseline cognitive assessment data are presented in Table 1 .

\section{Analysis of MRI data}

Structural images were acquired using a 3.0 Tesla magnetic resonance scanner (Philips). Borders of the anterior (head) and posterior (body and tail) hippocampus were traced manually (see Figure 1) on T1 weighted images using the ITK snap program, simultaneously monitoring the sagittal, coronal, and axial views according to standard published protocol (Watson et al., 1992). The hippocampus was traced in successive coronal slices in anterior to posterior direction using the alveus as the superior boundary and the white matter of the parahippocampal gyrus as the inferior boundary. The inferior temporal horn of the lateral ventricle was used as the lateral boundary and the ambient cistern the medial boundary. The boundary between hippocampal head and body was determined as the last slice on which the uncus was visible.

TABLE 1

Demographics

\begin{tabular}{lll}
\hline & Older $(n=21)$ & Young $(n=24)$ \\
\hline Age & $65.4 \pm 2.5$ & $25.3 \pm 2.8$ \\
Gender & $13(61.9 \%$ female $)$ & $12(50 \%)$ female \\
Race/ethnicity* & & \\
Hispanic & 0 & 1 \\
White & 14 & 12 \\
Black or African-American & 7 & 7 \\
Asian & 0 & 3 \\
Education in years & $15.5 \pm 3.3$ & $15.5 \pm 2.1$ \\
NART verbal IQ & $118.5 \pm 10.2$ & $112.9 \pm 8.4$ \\
NART performance IQ & $116.6 \pm 7.2$ & $112.6 \pm 5.9$ \\
WAIS voc & $52.6 \pm 11.8$ & $48.9 \pm 12.7$ \\
WTAR & $39.8 \pm 10.6$ & $38.8 \pm 8.2$ \\
Mattis DRS Total & $140.5 \pm 3.1$ & $141.5 \pm 2.2$ \\
\hline
\end{tabular}

Note: *Missing ethnicity data on one young subject.

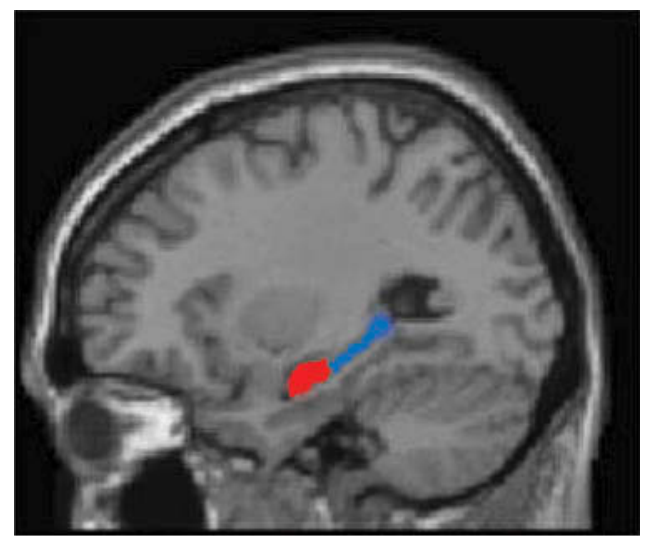

Figure 1. Illustration of anterior (red) and posterior (blue) hippocampus segmentations, here represented on a single subject's saggital T1 image. 
Posteriorly, the last slice of hippocampus was defined in the coronal plane as the slice $3 \mathrm{~mm}$ anterior to where the crura of the fornix separated from the hippocampus. In addition to the manual tracing, we performed automated segmentation of T1 images using FreeSurfer, version 5.1. Its delineation of the hippocampus has been validated in normal aging, mild cognitive impairment, and $\mathrm{AD}$ (Fischl et al., 2004; Gordon, Blazey, Benzinger, \& Head, 2013; Sánchez-Benavides et al., 2010). Our FreeSurfer extracted volumes are larger than manually extracted, and this has been reported in the literature. FreeSurfer volumes tend to be systematically larger than manual results, but as sensitive as manual tracing in detecting hippocampal volume differences (Shen et al., 2010). Hippocampal volume was adjusted for intracranial volume (ICV).

\section{fMRI data acquisition}

Functional images were acquired using a 3.0 Tesla magnetic resonance scanner (Philips) using a field echo echo-planar imaging (FE-EPI) sequence $(\mathrm{TE} / \mathrm{TR}=20 \mathrm{~ms} / 2000 \mathrm{~ms}$; flip angle $=72$ degrees; $112 \times 112$ matrix; in-plane voxel size $=2.0 \mathrm{~mm} \times$ $2.0 \mathrm{~mm}$; slice thickness $=3.0 \mathrm{~mm}$ (no gap); 37 transverse slices per volume), interleaved, in ascending order. Participants were scanned for 9.5 minutes, with instructions to rest and to keep eyes open for the duration of the scan. A structural image was also obtained (T1-weighted turbo field echo high resolution image with $\mathrm{TE} / \mathrm{TR}=$ $2.98 \mathrm{~ms} / 6.57 \mathrm{~ms}$; flip angle $=8$ degrees; $256 \times 256$ matrix; in-plane voxel size $=1.0 \mathrm{~mm} \times 1.0 \mathrm{~mm}$; slice thickness $=1.0 \mathrm{~mm}$ (no gap); 165 slices $)$.

\section{fMRI data processing}

Using the SPM5 software package, the functional data were motion-corrected and co-registered to the structural data, spatially normalized to the MNI template and band-pass filtered $(0.01 \mathrm{~Hz}<\mathrm{f}<0.08 \mathrm{~Hz})$.

To remove noise from nuisance variables, including, but not limited to, subject motion, we focused on voxels that have very low gray matter probability $(0<\mathrm{P}<.01)$, and subjected these voxels to a prinicipal components analysis, creating time-courses of PCs with eigenvalues $>.0001$ as nuisance regressors. A linear regression of time-series against nuisance regressors was performed and residuals computed. Time-series residualized in this manner are corrected for $\mathrm{WM} / \mathrm{CSF}$ contributions (motion scanner noise, etc.; Behzadi, Restom, Liau, \& Liu, 2007) and for the possible motion artifacts which may affect analysis (Power, Barnes, Snyder, Schlaggar, \& Petersen, 2012; Van Dijk, Sabuncu, \& Buckner, 2012). Data were then spatially smoothed with a Gaussian kernel of FWHM $6 \mathrm{~mm}$. We restricted all further analysis to voxels with gray matter probability $>.5$.

Seed locations were chosen in anterior and the posterior hippocampus, for both the left and right hemisphere (MNI coordinates: Right anterior 28, -10 , -22 ; left anterior $-28,-10,-22$; right posterior $25-370$; left posterior $-25-370$ ). Seeds were extracted as $3 \times 3 \times 3$ voxel cubes centered at the seed locations. Time-series for all seeds were calculated as the mean over all 27 voxels within a seed. For each seed, correlation maps were created by correlating each mean time course from each seed with the time courses from every other voxel in the brain. Then, one Fisher-Z transformed image per subject was created, quantifying the strength of the association between the seed location and every other voxel in the brain. Group averages of the Z-images were created for the anterior and posterior seeds for the young and old groups.

Young and older subjects were first examined separately. Group averages were created for the young and old groups for the most anterior and most posterior seeds. The pattern of connectivity of the anterior and posterior seeds in the young and old groups was examined visually at thresholds of $p<.001$ and voxel extent of 10 (Figure 2 visual representation). The same group average z-maps were examined with a stricter statistical threshold of $p<.00001$ to identify areas of particularly high connectivity to the hippocampal seed locations (see Figure 2 tables). This was done as reliability of functional connectivity fMRI across studies is higher the stronger the correlation is (Shehzad et al., 2009). Next, a two-factor analysis of variance was set up in SPM with factors age group (young/older) and seed (anterior/posterior). The two age groups were compared in respect to their anterior and posterior hippocampus connectivity, by examining the interaction between age group and hippocampal seed location (see Figure 3). We elaborated on this analysis in order to examine the functional connectivity of the cortical brain regions identified in this analysis to the entire longitudinal axis of the hippocampus. Cortical seeds were extracted $(3 \times 3 \times 3$ voxel cubes $)$ at the locations identified through the age group-byhippocampus-seed-location interactions, and we examined the connectivity of the cortical seeds to eight locations spanning the entire longitudinal axis of the hippocampus (MNI coordinates: Right hippocampus $28-10-22$ (most anterior); $30-14$ $-18 ; 30-18-16 ; 30-22-14 ; 30-26-12 ; 30-29$ 

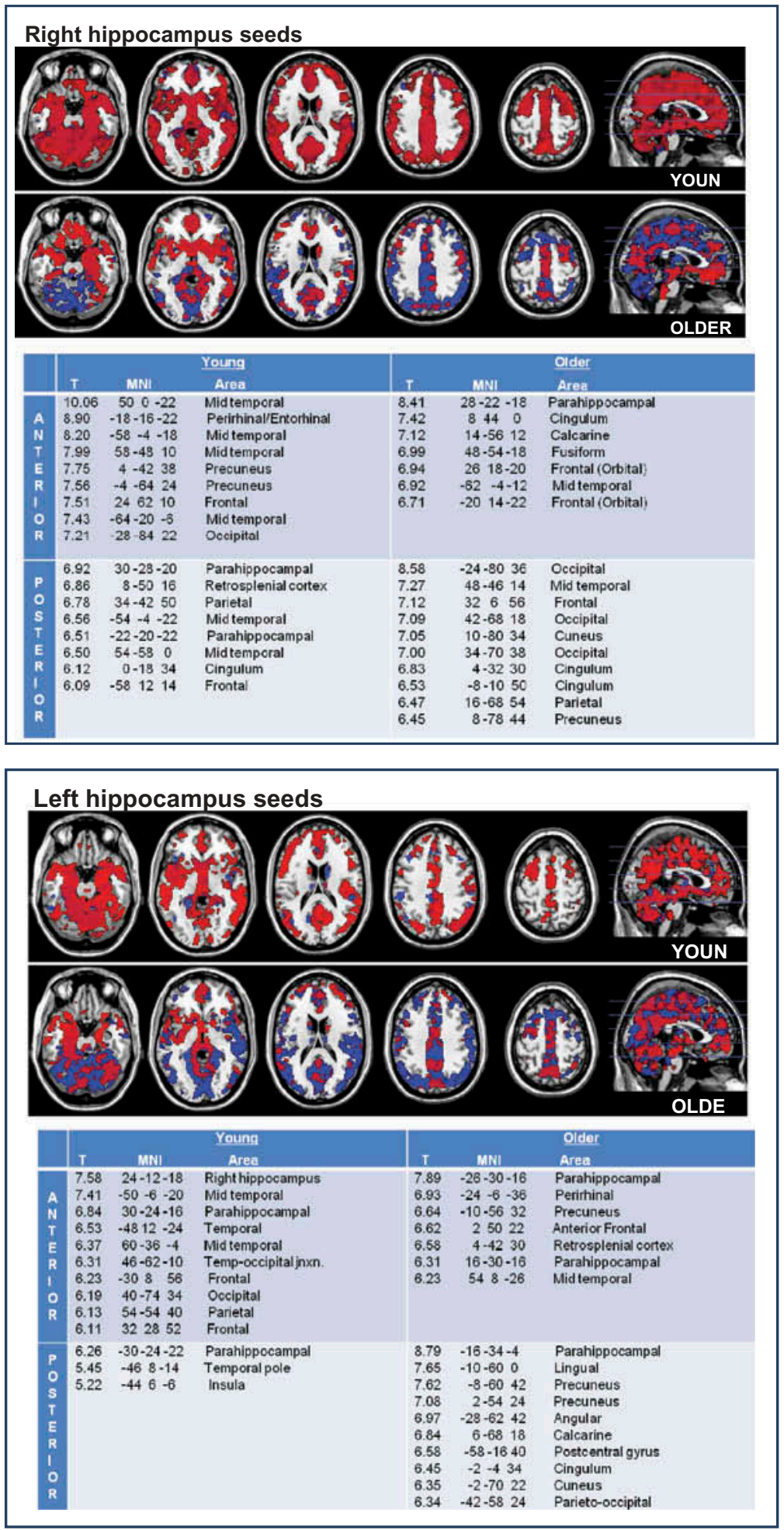

Figure 2. In younger individuals, anterior and posterior hippocampus are widely connected to other brain areas, with areas of highest connectivity noted in the table. In the older subjects, there is overall diminished functional connectivity of the hippocampus to cortical areas, but there was a relative increase in posterior hippocampal connectivity. Anterior hippocampus functional connectivity to cortical areas is noted in red, and posterior in blue. The table lists the brain regions most connected to the anterior and those most connected to the posterior hippocampus of young and older adults. 


\section{LEFT HIPPOCAMPUS SEED BASED ANALYSIS}

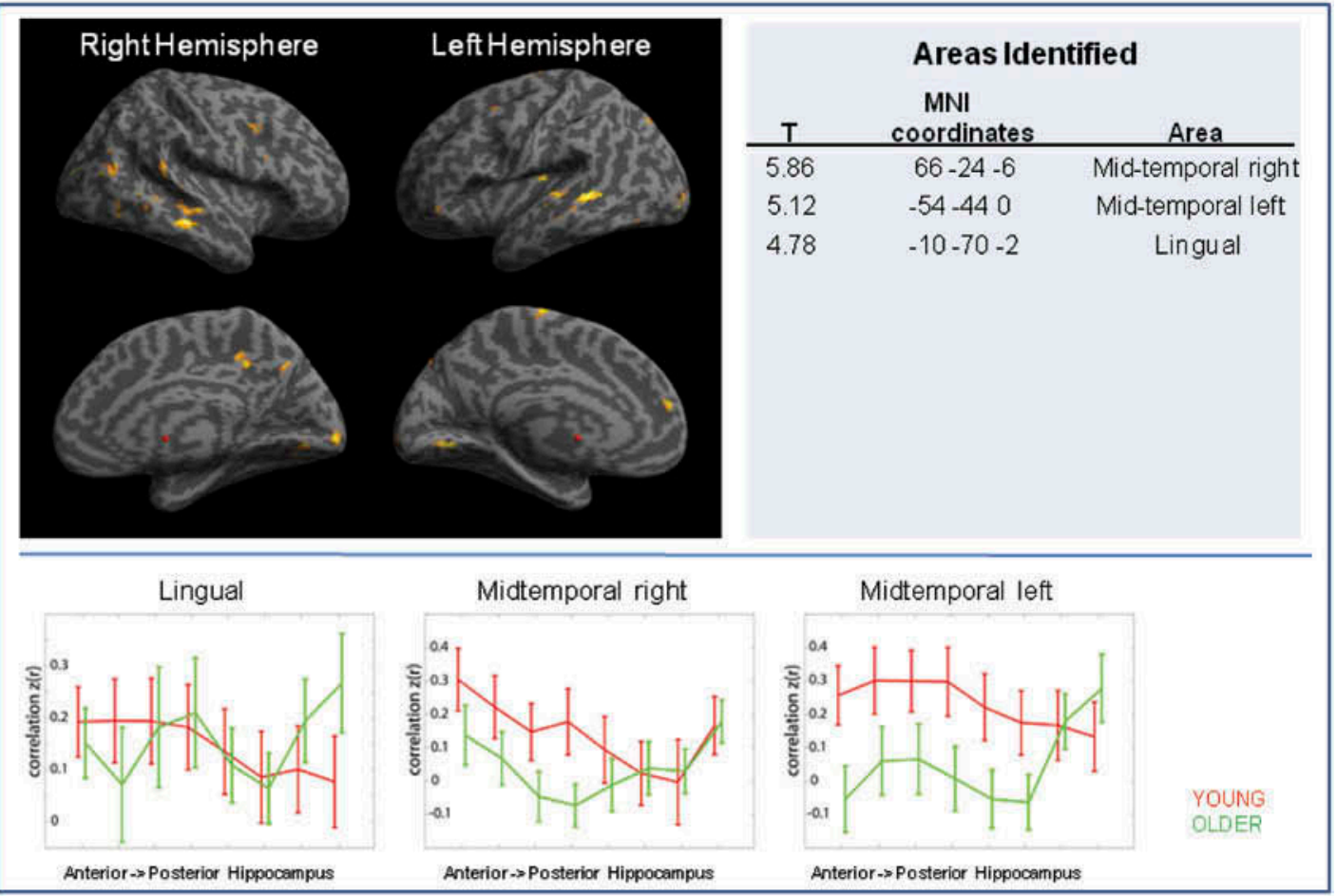

\section{RIGHT HIPPOCAMPUS SEED BASED ANALYSIS}
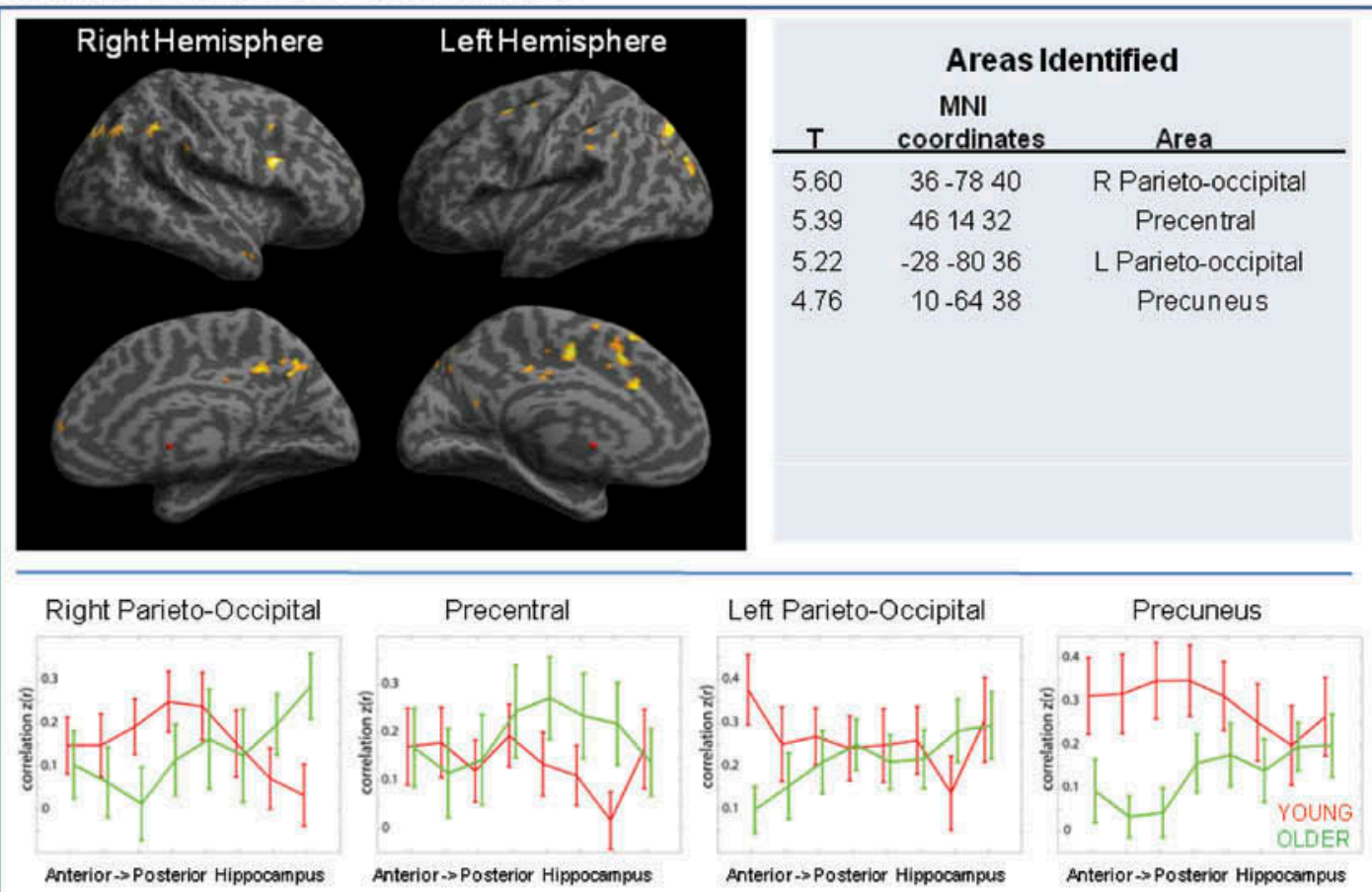

Figure 3. A set of brain regions is preferentially connected with the anterior hippocampus in the young but to the posterior hippocampus in the older subjects. This was observed both in left and right hippocampus seed-based analyses. There were no similar observations in the opposite direction (i.e., there were no areas that are preferentially connected to the posterior hippocampus in the young but to the anterior in older subjects) (Note: Results are overlaid on the inflated brain template provided in SPM 8). Largest cortical clusters identified are listed in the table. The graphs illustrate the strength of connectivity between the cortical regions and eight locations (see Methods) spanning the longitudinal axis of the hippocampus. As we move from anterior to posterior hippocampus, the strength of functional connectivity with the cortical nodes decreases moving posteriorly in young subjects, whereas it increases as we move posteriorly in older subjects. 
$-10 ; 30-33-6 ; 25-370$ (most posterior), left hippocampus -28-10-22 (most anterior); $-30-14$ $-18 ;-30-18-16 ;-30-22-14 ;-30-26-12 ;-30$ $-29-10 ;-30-33-6 ;-25-370$ (most posterior)). This sub-analysis elaborates on the major finding in Figure 3, and is not an example of circular analysis (Kriegeskorte, Simmons, Bellgowan, \& Baker, 2009).

\section{RESULTS}

\section{Hippocampal volume analysis}

Hippocampus volume did not differ significantly between young and older subjects (adjusted for ICV). LEFT: Young 0.1634 +/- 0.02136, older $0.1609+/-0.02066$; RIGHT: Young $0.1988+/-$ 0.03270 , older $0.2022+/-0.02073$. The proportion of anterior (aHPC) and posterior hippocampus (pHPC) also did not differ significantly between the two age groups (LEFT: aHPC young $1.21+/-0.19$ vs. older $1.27+/-0.26, p=.38$; $\mathrm{pHPC}$ young 0.84 $+/-0.13$ vs. older $0.81+/-0.15, p=.50$; RIGHT aHPC young $1.14+/-0.15$ vs. older $1.21+/-0.19$, $p=.18$; pHPC young $0.89+/-0.11$ vs. older $0.84+/-$ $0.13, p=.21)$. Automated FreeSurfer segmentation also showed that there was no statistically significant difference between hippocampal volumes of younger and older subjects (LEFT: Young 0.2975 +/-0.0245, older 0.2836 +/- 0.0301); RIGHT: Young 0.2998+/0.0290 , older $0.2894+/-0.0329)$. Although there was no significant difference, we found trends towards older subjects having a smaller left hippocampus and whole brain volume when compared to the younger subjects $(p=.135$ and $p=.074$, respectively). This was not observed for the right hippocampus $(p=.316)$.

\section{Anterior and posterior hippocampus connectivity in the young age group}

The right and left anterior hippocampus in younger subjects exhibited connectivity to the mid-temporal, and parts of the frontal (including orbital frontal), parietal, and occipital lobes (see Figure 2). In addition, the right anterior hippocampus had strong functional connectivity with the perirhinal/entorhinal and precuneus, whereas the left anterior hippocampus had strong functional connectivity with the parahippocampal gyrus. The left posterior hippocampus showed connectivity to the parahippocampal gyrus, the temporal pole, and the insula, whereas the right posterior hippocampus exhibited strongest functional connectivity with the parahippocampal region, retrosplenial cortex and parietal lobe (Figure 2).

\section{Older age group}

In older subjects, there was an overall diminished functional connectivity of the hippocampus to neocortical areas compared to the younger subjects, but there was a relative increase in connectivity to more posterior and postero-medial brain regions.

The older subjects' anterior hippocampi bilaterally demonstrated significant functional connectivity to the parahippocampal gyrus, anterior and inferior frontal and mid-temporal regions (see Figure 2). In addition, specific to the right anterior hippocampus, was a strong functional connectivity to the fusiform and calcarine cortices, and to the cingulum. Specific to the left anterior hippocampus, was a strong functional connectivity to the perirhinal, precuneus, and retrosplenial cortices.

The older subjects' posterior hippocampi showed strong connectivity to the cuneus, precuneus, and cingulum. In addition, the right posterior hippocampus showed strong connectivity to the occipital lobes, whereas the left posterior hippocampus to the parahippocampal, lingual, angular, and supramarginal gyrus and to the calcarine (Figure 2).

\section{Differential anterior/posterior hippocampus connectivity in young and older subjects}

Next, we examined the age group by seed location interaction to determine whether there were any significant differences between the two age groups in regard to connectivity of anterior and posterior hippocampus. The main finding was that a subset of brain areas had greater connectivity to the anterior hippocampus in young, but to the posterior in older subjects. Figure 3 shows brain regions identified as preferentially connected with the anterior hippocampus in the young but to the posterior hippocampus in the older subjects. The observation was made in analyses of both left and right hippocampus seed-based connectivity, but the pattern was different. There were no areas that were preferentially connected to the posterior hippocampus in the young but to the anterior in older subjects either in left or right hippocampusbased analysis. A contrast of posterior hippocampus connectivity between the young and older subjects confirmed that in older subjects there is a true increase in posterior hippocampus connectivity to several cortical 
regions (data not shown), rather than an artifact of the anterior hippocampus connectivity decline in the older adults. To elaborate on this finding, we examined the functional connectivity of the identified cortical regions to the entire longitudinal axis of the hippocampus rather than just to the anterior and posterior seed. To do so, we extracted seeds at the cortical areas identified through the interaction analysis and checked the connectivity of the cortical seeds across eight locations spanning the entire longitudinal axis of the hippocampus (see Figure 3).

\section{GENERAL DISCUSSION}

Connectivity of the anterior and posterior hippocampus with the neocortex was compared across age groups. Our study has several key findings: (1) anterior and posterior hippocampus show differences in functional connectivity to neocortical area, in both young and older subjects; (2) older subjects show an overall reduction in hippocampal-neocortical connectivity; (3) there is a relative increase in posterior hippocampusneocortex connectivity in older adults; and (4) a set of brain regions are preferentially connected with the anterior hippocampus in the young, but to the posterior hippocampus in the older subjects, suggesting an agerelated reorganization of hippocampal-neocortical connectivity, which may have implications for compensatory mechanisms that allow maintaining normal memory functions into older age.

Our study provides further support for existence of distinct circuits to which anterior and posterior regions of the human hippocampus belong, and our observations that in young subjects, the right anterior hippocampus had strong functional connectivity with the perirhinal/ entorhinal, and the posterior hippocampus showed connectivity to the parahippocampal gyrus, the temporal pole, the insula, and the retrosplenial cortex are consistent with the literature (Kahn et al., 2008; Manns \& Eichenbaum, 2006; Moser \& Moser, 1998; Suzuki \& Amaral, 1994).

The older adults show an overall reduction in the functional connectivity of the hippocampus to whole brain. This is consistent with the recent work by Geerligs et al., which shows across the board decrease in functional connectivity with age within networks supporting higher level cognitive functions, such as the default mode, cingulo-opercular and fronto-parietal control networks (Geerligs, Renken, Saliasi, Maurits, \& Lorist, 2014). The underpinnings of this kind of observations are likely changes in the structural brain networks as we age (Zhu et al., 2012).
In older adults, we also find a distinct set of areas connected to the anterior and the posterior hippocampus. A prominent difference is that the anterior hippocampus of older adults did not show diffuse connectivity to a number of brain areas, but rather a limited connectivity to the anterior/inferior frontal lobes, parahippocampal gyrus, and the inferior and mid-temporal gyrus (see Figure 2). The posterior hippocampus of older adults showed connectivity in particular to more superior parietal lobe and posterior regions of the brain in general, including precuneus, cuneus, and posterior cingulate, occipital and parietal lobes, and parts of the temporal lobes (see Figure 2). Furthermore, we demonstrated that a number of cortical areas which show a strong functional connectivity to the anterior hippocampus in the young subjects, have a strong connectivity to the posterior hippocampus in the older subjects. Sperling and colleagues demonstrated that stronger connectivity between the hippocampus and posteromedial regions during rest predicted better memory performance (Wang et al., 2010). An interpretation of our observation of more focused hippocampal connectivity to the posterior and posteromedial cortices in older adults may be consistent with greater effort in the older brain to maintain normal memory functions. The superior parietal lobe has been implicated in sensory-motor integration and active maintenance of an internal representation of one's own body (Wolpert, Goodbody, \& Husain, 1998). Aspects of the observed connectivity to these regions among the older adults may be related to differences in sensory-motor integration in older adults. Future work will address the underpinnings of the connectivity differences.

With aging, there is atrophy of the hippocampus, but the structure is not uniformly affected. For example, CA1 atrophies to some degree, but the dentate gyrus and CA3 may be more affected in normal aging (Mueller \& Weiner, 2009) and anterior-posterior hippocampus differences in atrophy pattern are noted both in normal aging and progression to Alzheimer's disease (Chen et al., 2010; Driscoll et al., 2009; Frisoni et al., 2008; Jack et al., 1997; Rajah et al., 2010; Whitwell et al., 2007). In our sample, we did not detect a significant difference between total hippocampal volume between young and older subjects, and the proportion of anterior and posterior segments in the two age groups was also not significantly different. This is likely due to the fact that subjects in our sample (age 60-68) are younger than subjects captured in some of the classic studies showing the age-related atrophy (for example, in Clifford Jack's 1997 study, average age was $79+/-6.7$; Driscoll 
et al., 2009, capture subjects 64-86 years of age; Chen et al., 2010, capture 55-83 year olds and Frisoni et al., 2008, average age was $74+/-5$ ). We did see a trend towards smaller left hippocampal volume in older subjects, which supports the argument that we are capturing a sample on the way to significant age-related atrophy, but not quite there yet.

We highlight some of the inherent limitations of the technique. The confidence in using resting state BOLD fMRI to investigate functional connectivity of brain regions in vivo in human subjects has increased based on accumulating evidence that the technique reflects underlying functional-anatomic correlation between brain regions (Arieli, Shoham, Hildesheim, \& Grinvald, 1995; Biswal, Yetkin, Haughton, \& Hyde, 1995; De Luca, Beckmann, De Stefano, Matthews, \& Smith, 2006; Greicius, Krasnow, Reiss, \& Menon, 2003; Kenet, Bibitchkov, Tsodyks, Grinvald, \& Arieli, 2003; Vincent et al., 2006). Resting state BOLD fMRI assumes sufficient constraint by anatomy to allow meaningful estimates of connectivity between brain regions (Van Dijk et al., 2010). Possible sources that contribute to the resting state functional correlations are monosynaptic and polysynaptic anatomic connectivity, anatomic connectivity to the same source, differences in synaptic efficiency across anatomically connected systems, and transient sources such as differences in synaptic efficiency reflecting recent experience (Fox \& Raichle, 2007). Thus, clearly this is not a direct measure of anatomic connectivity, but it has been shown to provide a great deal of indirect information about connectivity. The reliability of functional connectivity fMRI across studies is relatively high (Shehzad et al., 2009), which is reassuring. In particular, strongest correlations are the most reliable, and that is why we used a rather stringent statistical cut-off to look for regions which are most connected to the hippocampus. Finally, in functional imaging studies, atrophy is a concern when comparing young and aged adults, as spatial normalization steps can be a source of systematic error if there are significant volume differences (Krishnan, Slavin, Tran, Doraiswamy, \& Petrella, 2006).

Understanding hippocampus-neocortex functional integration/connectivity across the life span will help in identifying patterns of brain organization associated with normal memory functioning. Of particular relevance, is identifying age-specific hippocampal-neocortical connectivity, which may have implications for targeting therapies that supports normal memory functions in younger individuals as well as at an older age.

Original manuscript received 16 July 2014

Revised manuscript received 7 October 2014

First published online 30 October 2014

\section{REFERENCES}

Arieli, A., Shoham, D., Hildesheim, R., \& Grinvald, A. (1995). Coherent spatiotemporal patterns of ongoing activity revealed by real-time optical imaging coupled with single-unit recording in the cat visual cortex. Journal of Neurophysiology, 73(5), 2072-2093.

Behzadi, Y., Restom, K., Liau, J., \& Liu, T. T. (2007). A component based noise correction method (CompCor) for BOLD and perfusion based fMRI. Neurolmage, 37(1), 90-101. doi:10.1016/j.neuroimage.2007.04.042

Biswal, B., Yetkin, F. Z., Haughton, V. M., \& Hyde, J. S. (1995). Functional connectivity in the motor cortex of resting human brain using echo-planar MRI. Magnetic Resonance in Medicine, 34(4), 537-541. doi:10.1002/ mrm.1910340409

Chen, K. H., Chuah, L. Y., Sim, S. K., \& Chee, M. W. (2010). Hippocampal region-specific contributions to memory performance in normal elderly. Brain and Cognition, 72(3), 400-407. doi:10.1016/j.bandc.2009. 11.007 10.1016/j.bandc.2009.11.007

Dash, P. K., Hebert, A. E., \& Runyan, J. D. (2004). A unified theory for systems and cellular memory consolidation. Brain Research Reviews, 45(1), 30-37. doi:10.1016/j.brainresrev.2004.02.001.

De Luca, M., Beckmann, C. F., De Stefano, N., Matthews, P. M., \& Smith, S. M. (2006). fMRI resting state networks define distinct modes of long-distance interactions in the human brain. NeuroImage, 29(4), 1359-1367. doi:10.1016/j.neuroimage.2005.08.035

Driscoll, I., Davatzikos, C., An, Y., Wu, X., Shen, D., Kraut, M., \& Resnick, S. M. (2009). Longitudinal pattern of regional brain volume change differentiates normal aging from MCI. [Research Support, N.I.H., Extramural Research Support, N.I.H., Intramural]. Neurology, 72(22), 1906-1913. doi:10.1212/WNL.0b $013 \mathrm{e} 3181 \mathrm{a} 82634$

Fischl, B., van der Kouwe, A., Destrieux, C., Halgren, E., Segonne, F., Salat, D. H., ... Dale, A. M. (2004). Automatically parcellating the human cerebral cortex. [Research Support, Non-U.S. Gov't Research Support, U.S. Gov't, P.H.S.]. Cereb Cortex, 14(1), 11-22.

Fox, M. D., \& Raichle, M. E. (2007). Spontaneous fluctuations in brain activity observed with functional magnetic resonance imaging. Nature Reviews Neuroscience, 8(9), 700-711. doi:10.1038/nrn2201

Freesurfer (?.?) [Computer software]. http://surfer.nmr.mgh. harvard.edu/

Frisoni, G. B., Ganzola, R., Canu, E., Rub, U., Pizzini, F. B., Alessandrini, F., ... Thompson, P M. (2008). Mapping local hippocampal changes in Alzheimer's disease and normal ageing with MRI at 3 Tesla. Brain, 131(12), 3266-3276. doi:10.1093/brain/awn280 
Geerligs, L., Renken, R. J., Saliasi, E., Maurits, N. M., \& Lorist, M. M. (2014). A brain-wide study of age-related changes in functional connectivity. Cerebral Cortex, doi:10.1093/cercor/bhu012

Gordon, B. A., Blazey, T., Benzinger, T. L., \& Head, D. (2013). Effects of aging and Alzheimer's disease along the longitudinal axis of the hippocampus. [Randomized Controlled Trial Research Support, N.I.H., Extramural]. Journal of Alzheimer's Disease, 37(1), 41-50. doi:10.3233/JAD-130011

Greicius, M. D., Krasnow, B., Reiss, A. L., \& Menon, V. (2003). Functional connectivity in the resting brain: A network analysis of the default mode hypothesis. Proceedings of the National Academy of Sciences, 100(1), 253-258. doi:10.1073/pnas.0135058100 0135058100

Jack, C. R. Jr, Petersen, R. C., Xu, Y. C., Waring, S. C., O'Brien, P. C., Tangalos, E. G., ... Kokmen, E. (1997). Medial temporal atrophy on MRI in normal aging and very mild Alzheimer's disease. Neurology, 49(3), 786-794. doi:10.1212/WNL.49.3.786

Kahn, I., Andrews-Hanna, J. R., Vincent, J. L., Snyder, A. Z., \& Buckner, R. L. (2008). Distinct cortical anatomy linked to subregions of the medial temporal lobe revealed by intrinsic functional connectivity. Journal of Neurophysiology, 100(1), 129-139. doi:10. 1152/jn.00077.2008

Kenet, T., Bibitchkov, D., Tsodyks, M., Grinvald, A., \& Arieli, A. (2003). Spontaneously emerging cortical representations of visual attributes. Nature, 425(6961), 954-956. doi:10.1038/nature02078

Kriegeskorte, N., Simmons, W. K., Bellgowan, P. S., \& Baker, C. I. (2009). Circular analysis in systems neuroscience: The dangers of double dipping. Nature Neuroscience, 12(5), 535-540. doi:10.1038/nn.2303

Krishnan, S., Slavin, M. J., Tran, T. T., Doraiswamy, P. M., \& Petrella, J. R. (2006). Accuracy of spatial normalization of the hippocampus: Implications for fMRI research in memory disorders. NeuroImage, 31(2), 560-571. doi:10.1016/j.neuroimage.2005.12.061

Manns, J. R., \& Eichenbaum, H. (2006). Evolution of declarative memory. Hippocampus, 16(9), 795-808. doi:10.1002/hipo.20205

Marr, D. (1971). Simple memory: A theory for archicortex. Philosophical Transactions of the Royal Society B: Biological Sciences, 262(841), 23-81. doi:10.1098/ rstb.1971.0078

McClelland, J. L., McNaughton, B. L., \& O'Reilly, R. C. (1995). Why there are complementary learning systems in the hippocampus and neocortex: Insights from the successes and failures of connectionist models of learning and memory. Psychological Review, 102(3), 419-457. doi:10.1037/0033-295X.102.3.419

Moser, M. B., \& Moser, E. I. (1998). Functional differentiation in the hippocampus. Hippocampus, 8(6), 608-619. doi:10.1002/(SICI)1098-1063(1998)8:6<608: AID-HIPO3>3.0.CO;2-7

Mueller, S. G., \& Weiner, M. W. (2009). Selective effect of age, Apo e4, and Alzheimer's disease on hippocampal subfields. Hippocampus, 19(6), 558-564. doi:10.1002/ hipo. 20614

Nadel, L., \& Moscovitch, M. (1997). Memory consolidation, retrograde amnesia and the hippocampal complex. Current Opinion in Neurobiology, 7(2), 217-227. doi:10.1016/S0959-4388(97)80010-4 [pii
Poppenk, J., \& Moscovitch, M. (2011). A hippocampal marker of recollection memory ability among healthy young adults: Contributions of posterior and anterior segments. Neuron, 72(6), 931-937. doi:10.1016/j.neuron. 2011.10.014

Power, J. D., Barnes, K. A., Snyder, A. Z., Schlaggar, B. L., \& Petersen, S. E. (2012). Spurious but systematic correlations in functional connectivity MRI networks arise from subject motion. [Research Support, N.I.H., Extramural Research Support, Non-U.S. Gov't Research Support, U.S. Gov't, Non-P.H.S]. NeuroImage, 59(3), 2142-2154. doi:10.1016/ j.neuroimage.2011.10.018

Rajah, M. N., Kromas, M., Han, J. E., \& Pruessner, J. C. (2010). Group differences in anterior hippocampal volume and in the retrieval of spatial and temporal context memory in healthy young versus older adults. Neuropsychologia, 48(14), 4020-4030. doi:10.1016/j. neuropsychologia.2010.10.010

Sánchez-Benavides, G., Gómez-Ansón, B., Sainz, A., Vives, Y., Delfino, M., \& Peña-Casanova, J. (2010). Manual validation of FreeSurfer's automated hippocampal segmentation in normal aging, mild cognitive impairment, and Alzheimer Disease subjects. [Research Support, Non-U.S. Gov't]. Psychiatry Research: Neuroimaging, 181(3), 219-225. doi:10.1016/j. pscychresns.2009.10.011

Shehzad, Z., Kelly, A. M., Reiss, P. T., Gee, D. G., Gotimer, K., Uddin, L. Q., ... Milham, M P. (2009). The resting brain: Unconstrained yet reliable. Cerebral Cortex, 19(10), 2209-2229. doi:10.1093/cercor/bhn256

Shen, L., Saykin, A. J., Kim, S., Firpi, H. A., West, J. D., Risacher, S. L., ... Flashman, L A. (2010). Comparison of manual and automated determination of hippocampal volumes in MCI and early AD. [Comparative Study Research Support, N.I.H., Extramural Research Support, Non-U.S. Gov't]. Brain Imaging and Behavior, 4(1), 86-95. doi:10.1007/s11682-010-9088-x

Small, S. A., Schobel, S. A., Buxton, R. B., Witter, M. P., \& Barnes, C. A. (2011). A pathophysiological framework of hippocampal dysfunction in ageing and disease. Nature Reviews Neuroscience, 12(10), 585-601. doi:10.1038/nrn3085 nrn3085

SPM (5) [Computer software]. http://www.fil.ion.ucl.ac.uk/ spm/software/

Squire, L. R., \& Alvarez, P. (1995). Retrograde amnesia and memory consolidation: A neurobiological perspective. Current Opinion in Neurobiology, 5(2), 169-177.

Suzuki, W. A., \& Amaral, D. G. (1994). Perirhinal and parahippocampal cortices of the macaque monkey: Cortical afferents. The Journal of Comparative Neurology, 350(4), 497-533. doi:10.1002/cne.903500402

Teyler, T. J., \& DiScenna, P. (1985). The role of hippocampus in memory: A hypothesis. Neuroscience \& Biobehavioral Reviews, 9(3), 377-389. doi:10.1016/ 0149-7634(85)90016-8

Van Dijk, K. R., Hedden, T., Venkataraman, A., Evans, K. C., Lazar, S. W., \& Buckner, R. L. (2010). Intrinsic functional connectivity as a tool for human connectomics: Theory, properties, and optimization. Journal of Neurophysiology, 103(1), 297-321. doi:10.1152/jn.00783.2009

Van Dijk, K. R., Sabuncu, M. R., \& Buckner, R. L. (2012). The influence of head motion on intrinsic functional 
connectivity MRI. [Research Support, N.I.H., Extramural Research Support, Non-U.S. Gov't]. NeuroImage, 59(1), 431-438. doi:10.1016/j.neuroimage.2011.07.044

Vincent, J. L., Snyder, A. Z., Fox, M. D., Shannon, B. J., Andrews, J. R., Raichle, M. E., \& Buckner, R. L. (2006). Coherent spontaneous activity identifies a hippocampal-parietal memory network. Journal of Neurophysiology, 96(6), 3517-3531. doi:10.1152/ jn.00048.2006

Wang, L., Laviolette, P., O’Keefe, K., Putcha, D., Bakkour, A., Van Dijk, K. R., ... Sperling, R A. (2010). Intrinsic connectivity between the hippocampus and posteromedial cortex predicts memory performance in cognitively intact older individuals. NeuroImage, 51(2), 910-917. doi:10.1016/j.neuroimage.2010.02.046

Watson, C., Andermann, F., Gloor, P., Jones-Gotman, M., Peters, T., Evans, A., ... Leroux, G. (1992). Anatomic basis of amygdaloid and hippocampal volume measurement by magnetic resonance imaging. Neurology, 42(9), 1743-1750. doi:10.1212/WNL.42.9.1743

Whitwell, J. L., Petersen, R. C., Negash, S., Weigand, S. D., Kantarci, K., Ivnik, R. J., ... Jack, C R., Jr. (2007). Patterns of atrophy differ among specific subtypes of mild cognitive impairment. Archives of Neurology, 64(8), 1130-1138. doi:10.1001/archneur.64.8.1130

Wolpert, D. M., Goodbody, S. J., \& Husain, M. (1998). Maintaining internal representations: The role of the human superior parietal lobe. [Case Reports Research Support, Non-U.S. Gov't]. Nature Neuroscience, 1(6), 529-533. doi:10.1038/2245

Zhu, W., Wen, W., He, Y., Xia, A., Anstey, K. J., \& Sachdev, P. (2012). Changing topological patterns in normal aging using large-scale structural networks. [Comparative Study Research Support, Non-U.S. Gov't]. Neurobiology of Aging, 33(5), 899-913. doi:10.1016/j.neurobiolaging.2010.06.022 
Copyright of Cognitive Neuroscience is the property of Psychology Press (UK) and its content may not be copied or emailed to multiple sites or posted to a listserv without the copyright holder's express written permission. However, users may print, download, or email articles for individual use. 